\title{
Un taller de simulaciones: Fathom, GeoGebra y Excel para resolver problemas controversiales de probabilidad
}

\author{
Greivin Ramírez Arce. \\ gramirez@itcr.ac.cr \\ Instituto Tecnológico de Costa Rica
}

\section{Resumen.}

El taller pretende utilizar la simulación, en Fathom, GeoGebra y Excel con el fin de desarrollar el pensamiento instintivo y confrontarlo con el pensamiento analítico en el estudio de las distribuciones y probabilidades condicionales, y que lo anterior ayude a resolver problemas considerados controversiales de probabilidad. Los participantes trabajarán con actividades guiadas, podrán apreciar la riqueza didáctica de la simulación hecha con paquetes dinámicos y comparar las potencialidades que cada uno ofrece en la solución de diversos problemas.

Palabras clave: Simulación, Probabilidad, Fathom, GeoGebra y Excel.

\section{Abstract}

The workshop will use simulation in Fathom, GeoGebra and Excel to compare instinctive thinking to analytical thinking in the study of probability distributions and conditional probability, with the goal of improving problem solving in non-intuitive probability problems. Participants will work with guided activities, will acquire an appreciation of the richness of the simulation of dynamic packages, and will compare the potential offered by each of the various tools in solving problems.

Keywords: Simulation, Probability, Fathom, GeoGebra y Excel.

\subsection{Introducción}

Rossman (1995) citado por North, Scheiber y Ottaviani (2010) indica que, la literatura sobre educación estadística debe ser una demostración de que las estadísticas ideales se deben enseñar utilizando un enfoque basado en datos, con datos reales para enfatizar los principios y procedimientos estadísticos, en lugar del enfoque tradicional teórico donde el énfasis está simplemente en la identificación de la fórmula correcta y la realización de un cálculo. La formación de profesionales en matemática y estadística presenta grandes desafíos y uno de ellos es para los educadores la integración de la tecnología para optimizar la calidad de la enseñanza.

La simulación computacional es una alternativa en el estudio de la estocástica; ya que como lo refiere Erickson (2010), parece interesante ver como un programa que logre la simulación orientada de datos y dinámicos, puede 
guiar o interferir en mejor entendimiento matemático de conceptos tales como: valores esperados, variabilidad, cálculo de probabilidades y forma de las distribuciones desde su construcción en la solución de problemas.

Al enseñar Probabilidad y Estadística debe considerarse el desarrollo cognitivo del estudiante, puesto que en dichos campos, algunas veces, se trata con ideas abstractas y no tan relacionadas con su experiencia directa; además, debe hacerse a partir de situaciones prácticas y cotidianas, mediante el empleo de proyectos y asignaciones que favorezcan su comprensión según Batanero y Godino (2001). Es necesario plantear actividades que estimulen la experimentación, el desarrollo de conjeturas y la búsqueda de explicaciones en un ambiente donde, en la medida de lo posible, se promueva el uso de la tecnología en procesos de representación, exploración y análisis de la información que resulta ser un componente importante en el desarrollo del pensamiento estadístico.

\subsection{Objetivo del taller}

El taller busca colaborar con el impacto de la simulación computacional, hecha con Fathom, GeoGebra y Excel, en la solución de problemas difíciles de resolver con el formalismo estocástico.

\subsection{Justificación de pertinencia e interés del taller}

Inzunsa (2006) resume el éxito de los estudiantes al usar la simulación computacional (sugerida por Shaughnessy, 1992; Burrill, 2002; Sánchez, 2002; Lipson, 2002):

Los estudiantes encuentran sentido a la resolución de problemas de distribuciones mediante la simulación en Fathom una vez que se apropiaron de los recursos del software y después de haber abordado algunas actividades. Son capaces de construir por ellos mismos las distribuciones, generando las poblaciones, tomando muestras, definiendo estadísticos y calculado sus probabilidades. (p. 215)

GeoGebra, por otro lado, ha demostrado ser una herramienta con un potencial didáctico para el cumplimiento de objetivos didácticos, pero además provee un ambiente que estimula al estudiante para que formule hipótesis, según Ferrerira y otros (2009).

Al respecto, el Ministerio de Educación Pública de Costa Rica (2005) refiere que "es conveniente que se parta de lo concreto, en los temas que es posible, estimular al estudiante, para que empiece a crear sus propias estrategias y a resolver problemas en forma autónoma, sin tener que recurrir a recetas preestablecidas".

Todo esto justifica lo fundamental que es una culturalización de los estudiantes en procesos estocásticos a través de la simulación.

\subsection{Plan y metodología del taller}

El taller se dividirá en tres etapas:

Primera etapa: El uso de simulación en la resolución de problemas. Etapa introductoria donde se les habla a los participantes de la importancia de incluir en el aula herramientas tecnológicas, esto con el fin de poder utilizar 
la simulación para mejorar la enseñanza aprendizaje de la estocástica.

Segunda etapa: problemas guiados con procesos de simulación. Se resolverán dos actividades guiadas, una en Fathom y otra en GeoGebra, con el fin de que se familiaricen con los paquetes en la resolución de los problemas 1 y 2.

Problema 1. Frecuencias relativas y absolutas (Fathom). Al lanzar una moneda justa, ¿cuál de los siguientes eventos considera que es más probable?

( ) Obtener dos escudos en cuatro intentos

( ) Obtener 50 escudos en 100 intentos

( ) Los dos anteriores son igualmente probables

Una propuesta de algoritmo de simulación en Fathom es la siguiente:

1. Se construye una moneda convencional con caras Corona (C) y Escudo (E).

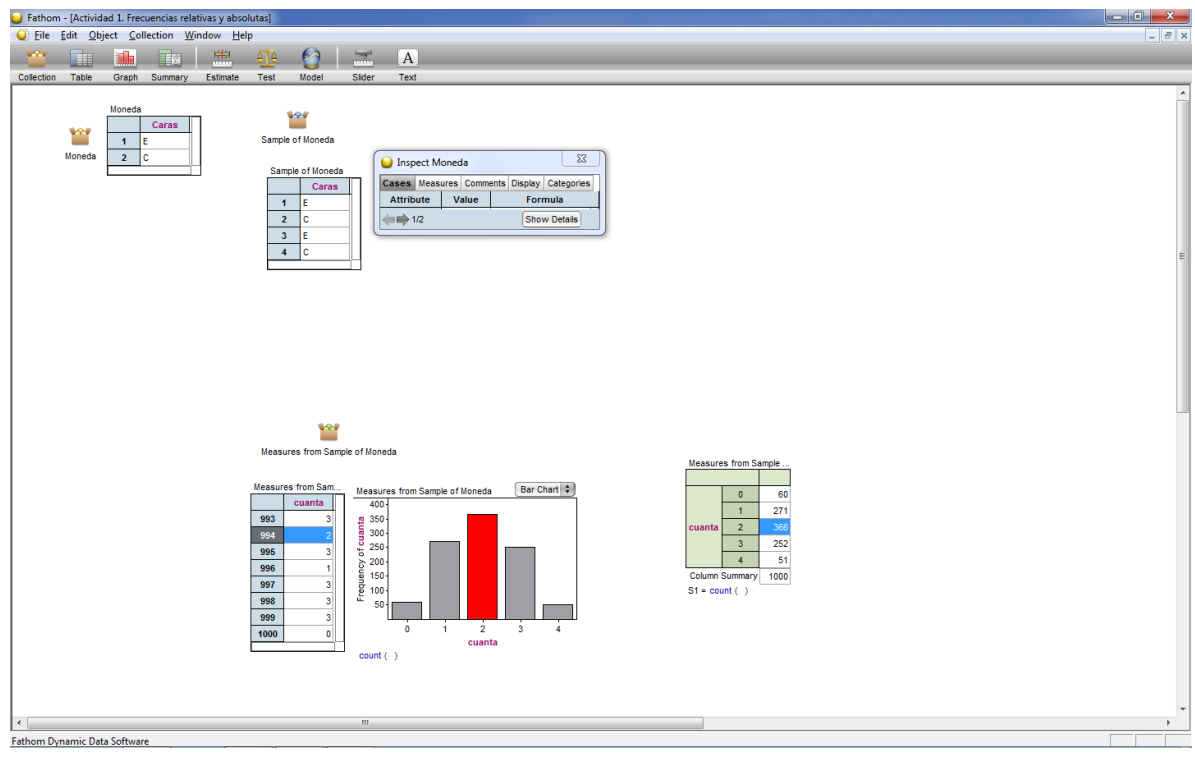

2. Se lanza la moneda cuatro veces y se cuenta las veces que ocurrió escudo.

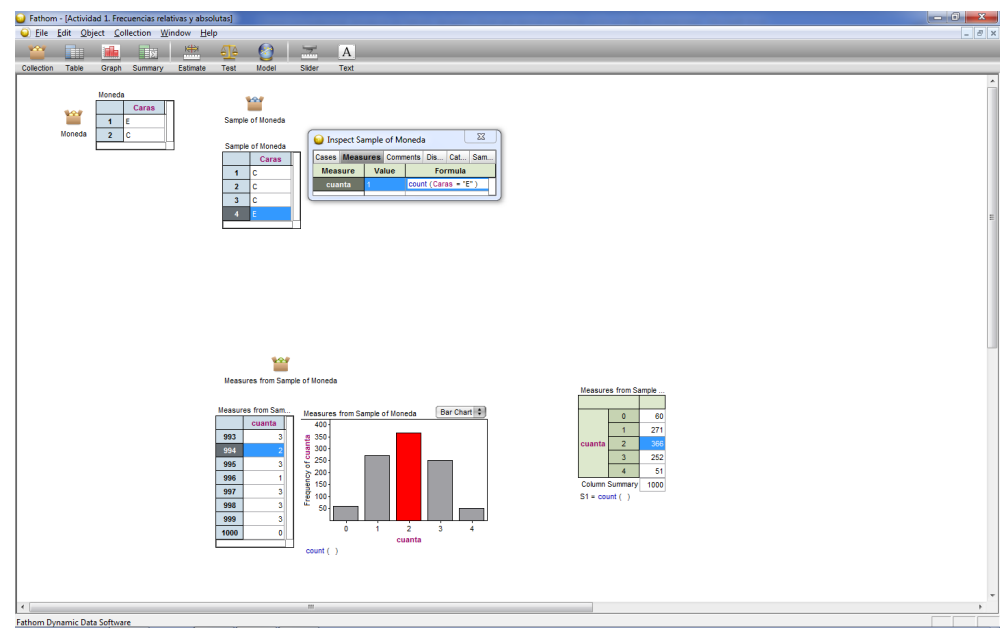

Un taller de simulaciones: Fathom, GeoGebra y Excel para resolver problemas controversiales de probabilidad. Greivin Ramírez Arce. Derechos Reservados (c) 2012 Revista digital Matemática, Educación e Internet (www.tec-digital.itcr.ac.cr/revistamatematica/) 
3. Se repite el experimento muchas veces (se recomiendan 1000) y se construye un diagrama de barras con las frecuencias absolutas de la cantidad de escudos obtenidos.

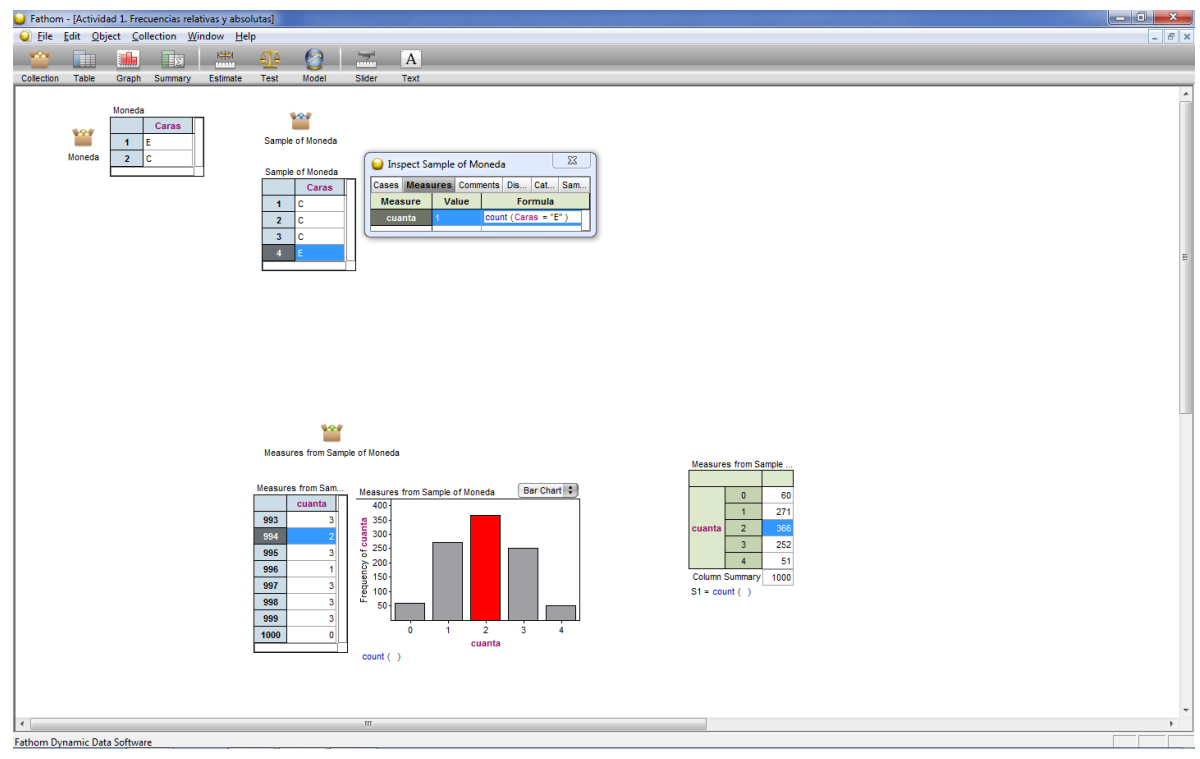

4. Se construye una tabla de frecuencias absolutas para determinar el número de veces en los que se obtuvo dos escudos de los 1000 experimentos realizados.

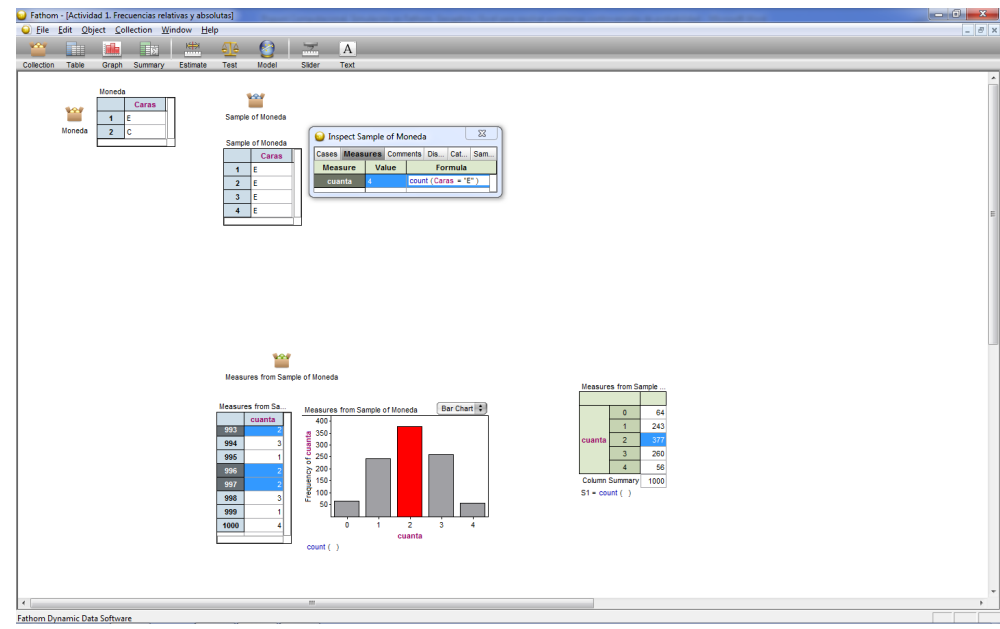

5. La probabilidad de obtener dos escudos en cuatro intentos fue de $\frac{377}{1000}=0.377$

6. Se repite el algoritmo del paso a. al e. para determinar el número de escudos que se obtiene al lanzar la moneda 100 veces. En un ejemplo particular, donde se repite el experimento 1000 ocasiones, la probabilidad de obtener 50 escudos en 100 intentos fue de $\frac{96}{1000}=0.096$ 


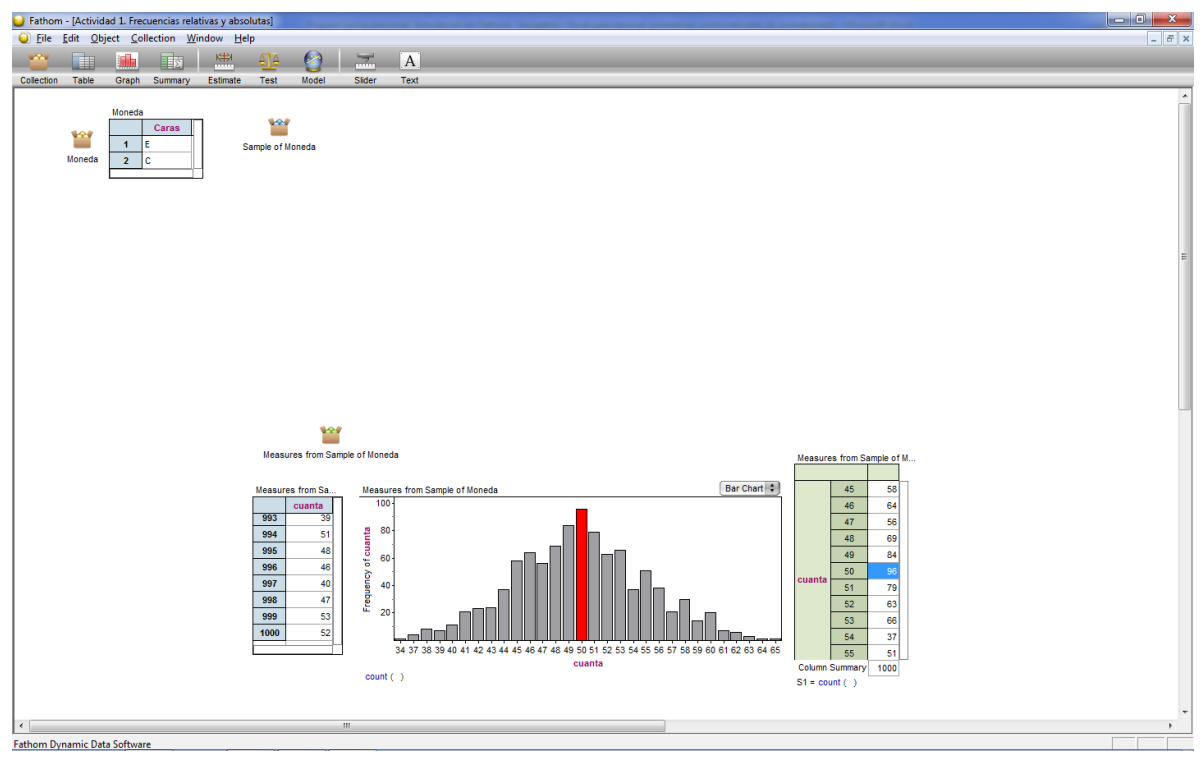

En el gráfico del punto c, donde se lanza la moneda cuatro veces en muchas ocasiones, se puede observar que la variabilidad es menor que el gráfico del punto $\mathrm{f}$, donde se lanza la moneda cincuenta veces en la misma cantidad de ocasiones. Razón por la cual es más probable obtener dos escudos en cuatro intentos, que cincuenta en cien intentos.

Problema 2. Problema del cáncer (GeoGebra) (en Shaughnessy, 1992). La probabilidad para una mujer de tener cáncer de mama sin haber presentado síntomas previos es de $0.8 \%$. Si tiene cáncer y se realiza la mamografía, la probabilidad de salir positiva es del $90 \%$, pero el $7 \%$ de mujeres sanas dan positivo en este examen. Suponga que una mujer decide hacerse una mamografía y el resultado es positivo, ¿cuál es la probabilidad de que la mujer tenga cáncer?

Una propuesta de algoritmo de simulación en GeoGebra es la siguiente:

1. Se obtienen 1000 números aleatorios entre 0 y 1 que representarán a 1000 mujeres cualesquiera (se sugiere tomar mayor número de mujeres si así lo desea):

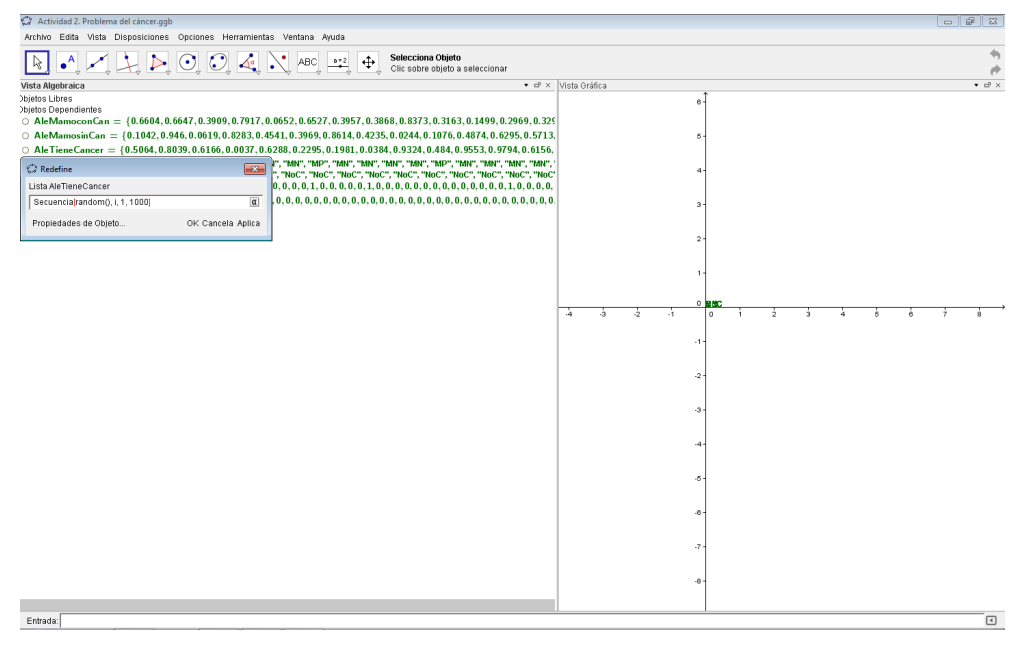

2. De estos 1000 valores los que sean menores que el $0.8 \%$ (0.008) serán las mujeres que tienen cáncer sin haber presentado síntomas previos, las demás $(99.2 \%)$ no tienen cáncer: 


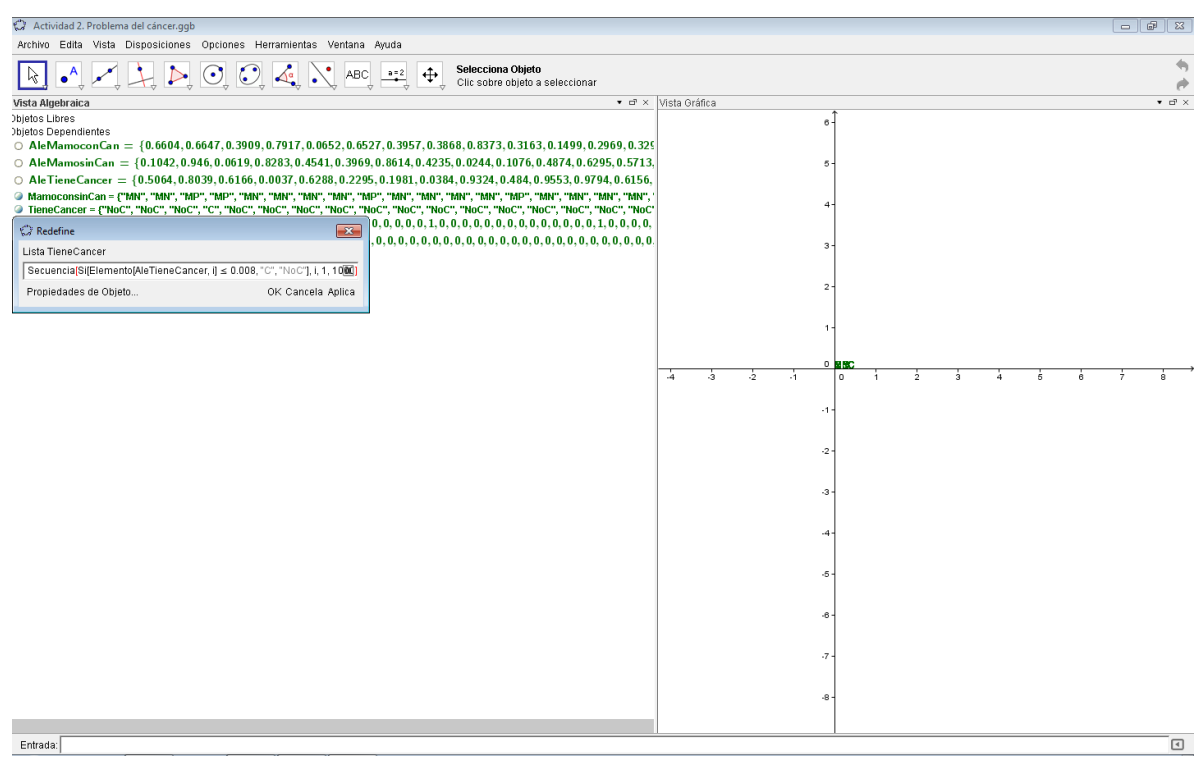

3. Con la idea intuitiva de un árbol,

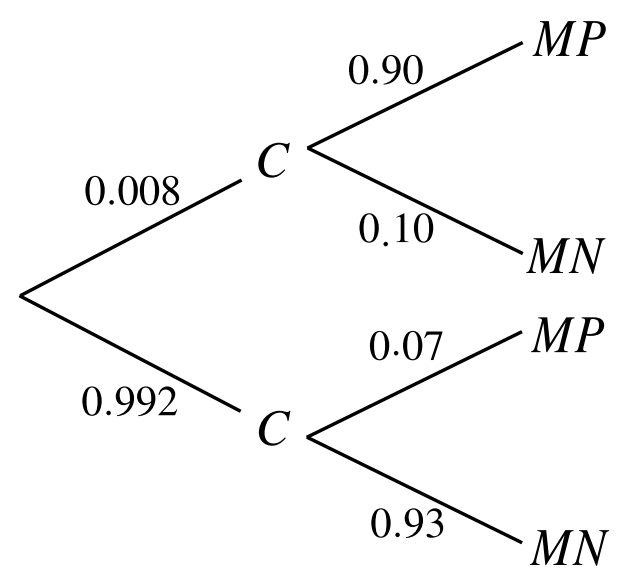

(a) Se obtienen 1000 números aleatorios entre 0 y 1 que serán las mujeres que cumplen la condición de estar sanas. De estos valores, los que sean menores que 7\% (0.07) serán las mujeres que se realizan la mamografía y resulta positiva, para las demás, su mamografía es negativa (93\%).

(b) Se obtienen 1000 números aleatorios entre 0 y 1 que serán las mujeres que cumplen la condición de tener cáncer. De estos valores, los que sean menores que 10\% (0.1) serán las mujeres que se realizan la mamografía y resulta negativa, para las demás, su mamografía resultó positiva (90\%). 


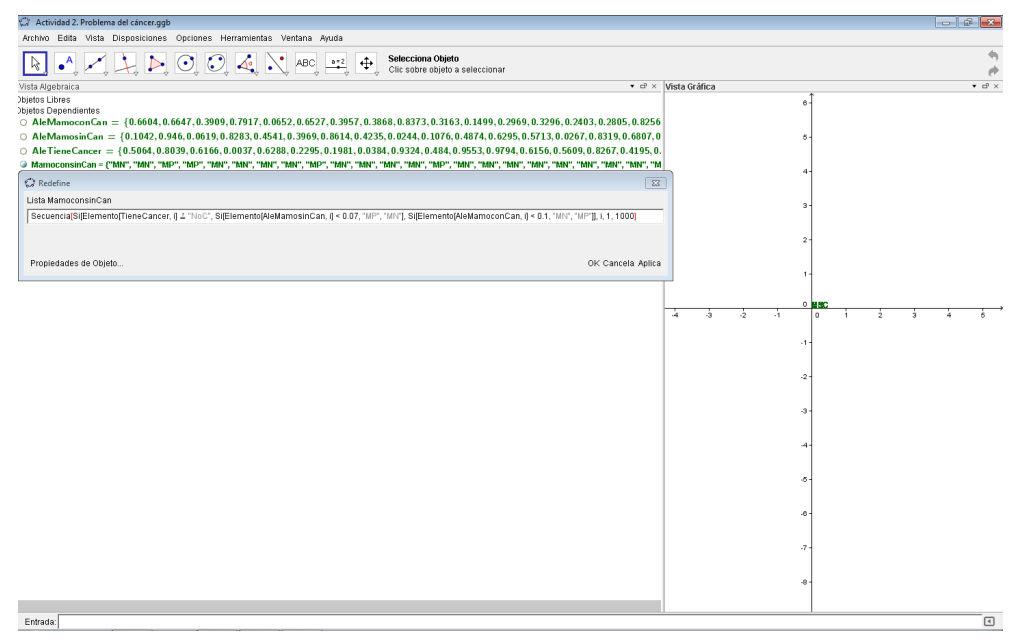

4. Interesa contar de las 1000 mujeres que se realizan la prueba, cuántas resultaron con mamografía positiva:

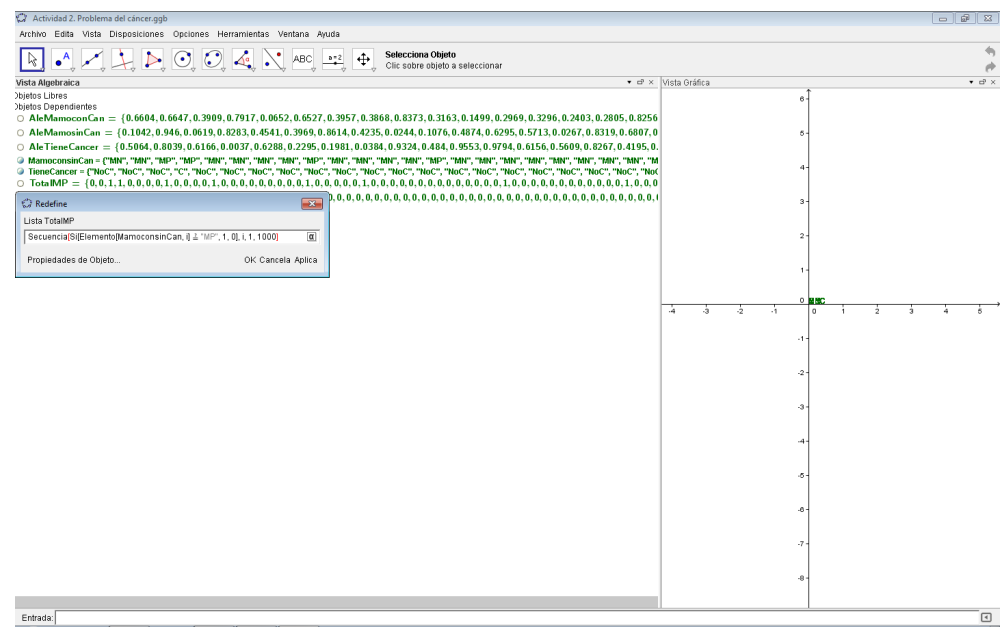

5. También interesa determinar de las 1000 mujeres, cuántas resultaron con cáncer y su mamografía fue positiva:

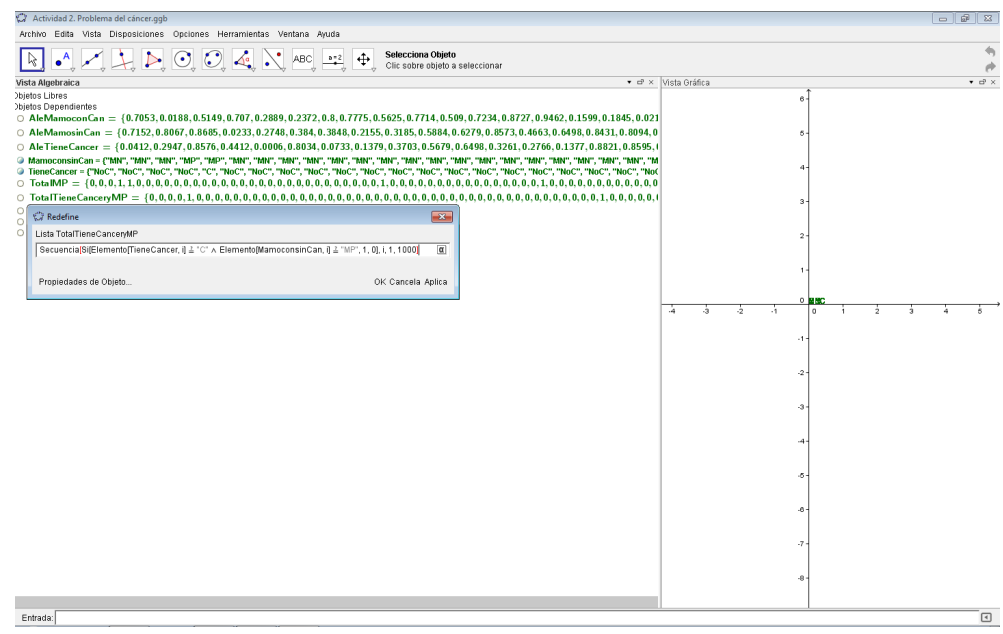


6. Se obtiene el cociente entre las mujeres que resultaron con cáncer y su mamografía fue positiva sobre el total de mujeres cuya mamografía fue positiva:
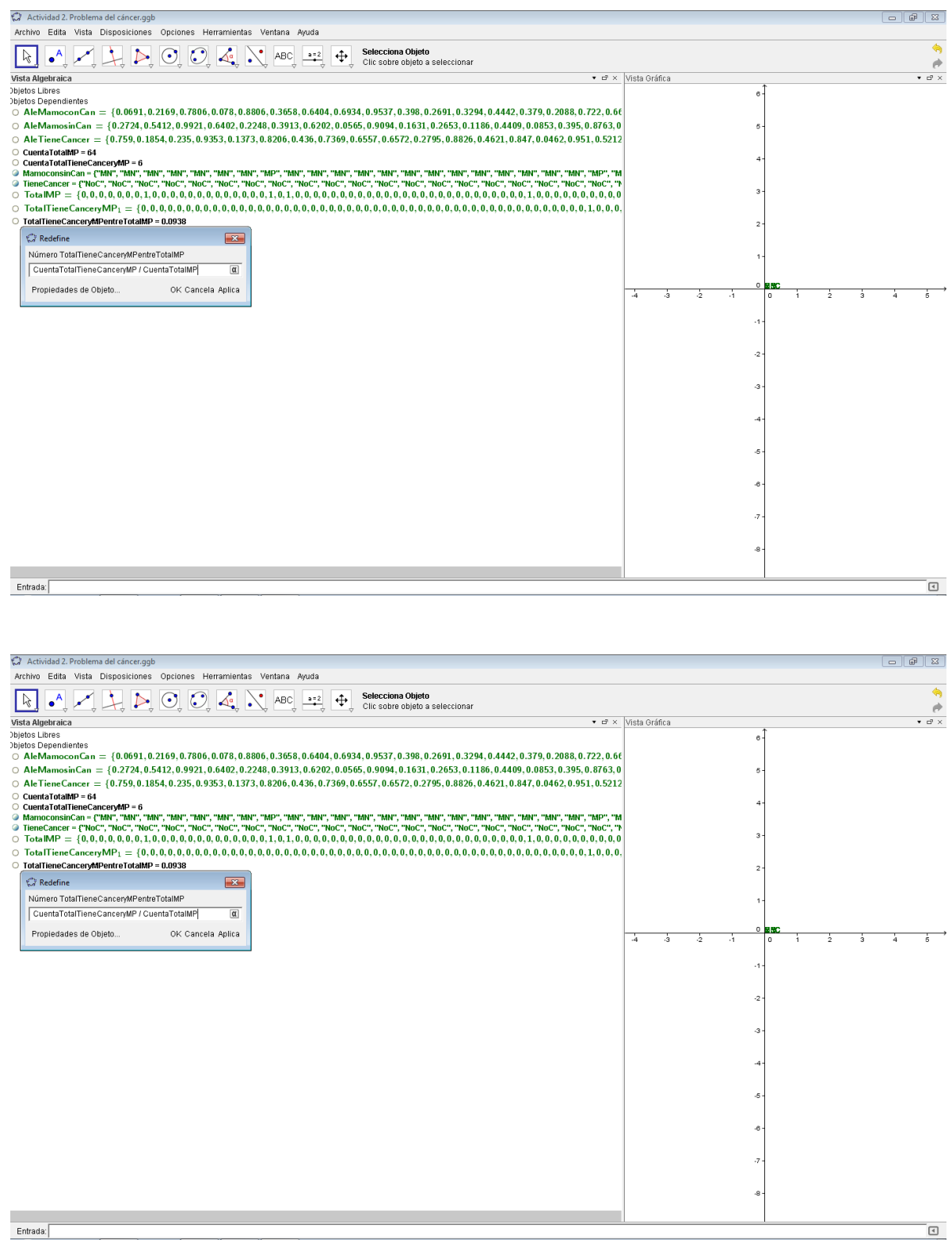

\section{Problemas complementacios para etapa 2.}

Problema 3. Dilama de Monty. En un concurso, un presentador le ofrece al concursante la posibilidad de elegir entre tres puertas, atrás de dos de ellas hay una cabra y en la otra se encuentra un auto. El concursante elige una puerta, y de forma inmediata el presentador abre dentro de las otras dos opciones aquella que tiene una cabra y le ofrece al concursante la posibilidad de cambiar su escogencia original. ¿Será bueno cambiar de puerta o quedarse con la selección original?

Problema 4. Problema del taxi (en Shaughnessy, 1992) (GeoGebra) Una cierta noche un taxi se ve involucrado en un accidente en la que pega y se escapa. En la ciudad operan dos compañías, la de los taxis verdes con el $85 \%$ y la de los azules con el 15\%. Un observador de la escena identifica al taxi que se escapó como un taxi azul. Este observador fue probado bajo condiciones normales de visibilidad e hizo una correcta identificación del color en 
80\% de los casos. ¿Cuál es la probabilidad de que el taxi fugado sea azul y no verde?

Tercera etapa: problemas abiertos con procesos de simulación.

Se resolverán los problemas 5 y 6 con Excel y Fathom respectivamente sin tener una guía formal, sino que sean los participantes que desarrollen las etapas del proceso de simulación con cada paquete.

Problema 5. El fenómeno de Falk (en Shaughnessy, 1992) (Excel). Una caja tiene en su interior tres bolas rojas $\mathrm{y}$ tres bolas azules. Se extraen dos bolas sin reemplazar la primera.

- ¿Cuál es la probabilidad de que la segunda bola sea roja dado que la primera fue roja?

- ¿Cuál es la probabilidad de que la primera bola sea roja, dado que la segunda es roja?

Para simular estos dos problemas se supone que las bolas numeradas del 1 al 3 son rojas y del 4 al 6 son azules. Aunque las bolas son indistinguibles cuando son del mismo color, se hará solo para facilitar el proceso de simulación.

Pregunta 1. Una propuesta de algoritmo de simulación en Excel es la siguiente:

1. Se define una primera columna en donde se obtiene la extracción de la primera bola. Sabemos que la primera bola es roja. Así que se realizan 1000 extracciones de bolas donde cada una será un número aleatorio entre 1 y 3 (con garantía es roja).

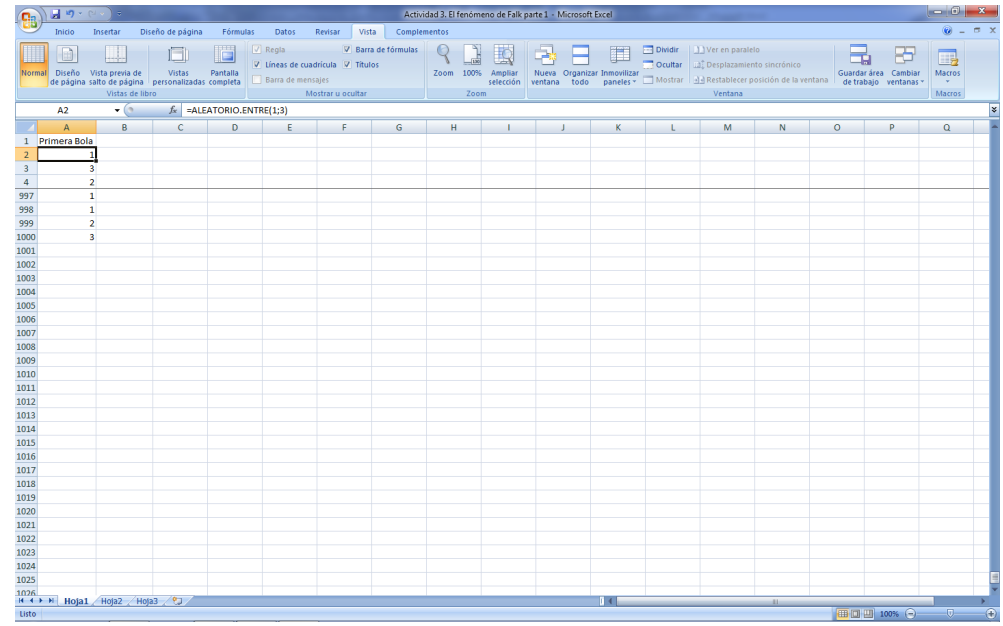

2. Dado que es sin reemplazo, en la segunda columna se debe extraer una bola entre las cinco opciones que quedan en la caja. Se tomará un número aleatorio entre 1 y 6 menos el valor extraído en la primera bola. 


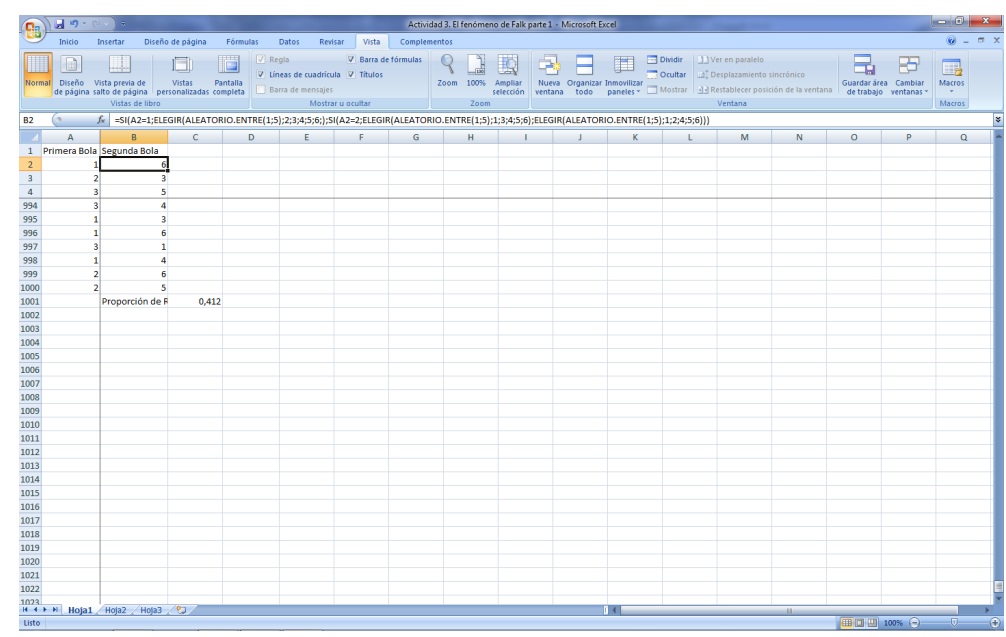

3. Por último se cuenta la cantidad de veces que la bola es roja (menor o igual a 3) en la segunda extracción de las 1000 realizadas.

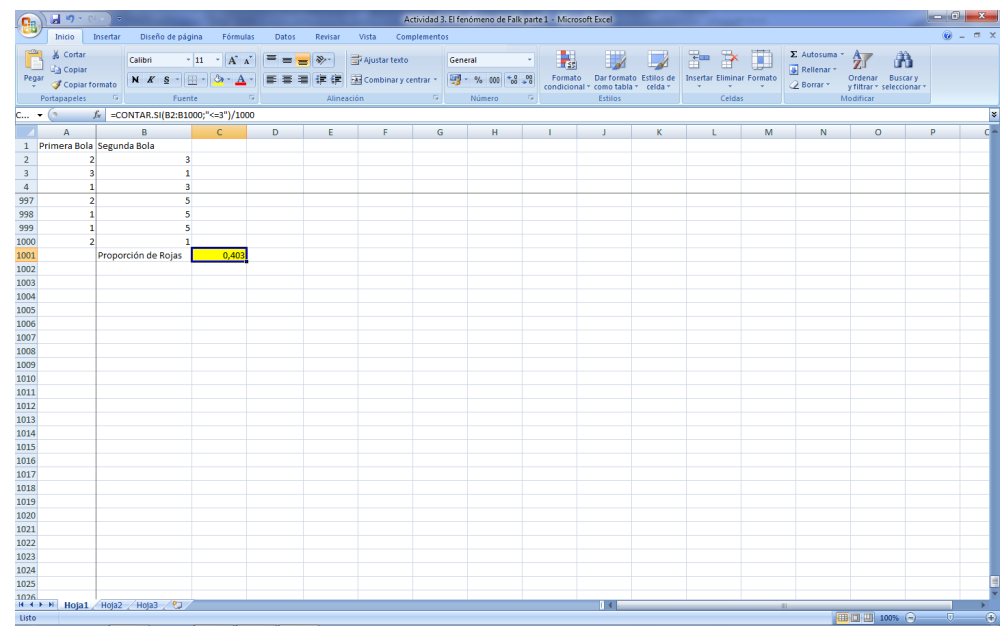

Pregunta 2. Una propuesta de algoritmo de simulación en Excel es la siguiente:

1. Se define la primera columna en donde se obtiene la extracción de la primera bola. Inicialmente se tienen en la caja 6 bolas, así que se realizan 1000 extracciones de bolas donde cada una será un número aleatorio entre 1 y 6 (podría ocurrir cualquier color): 


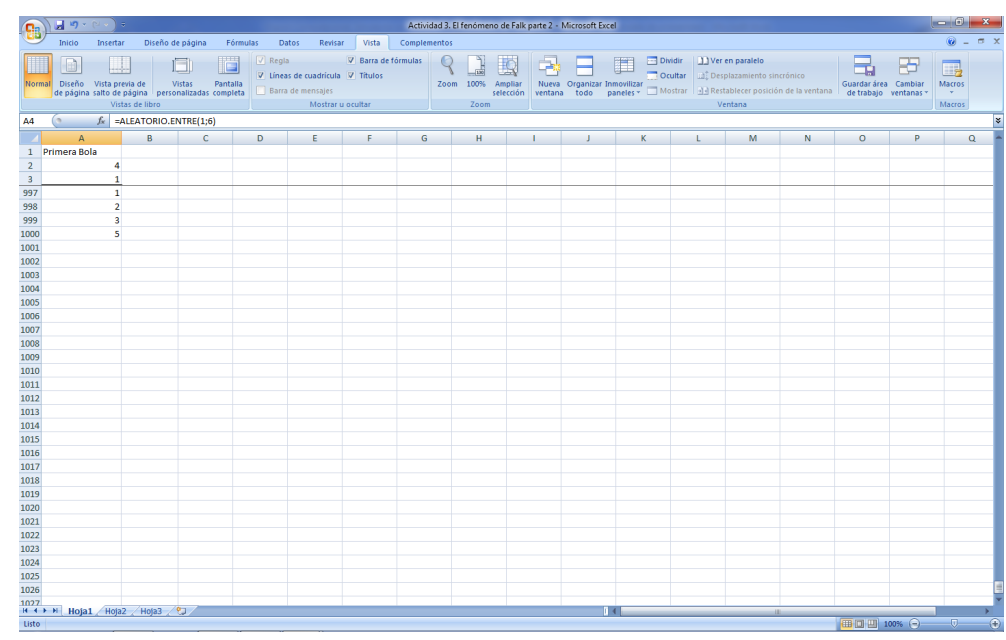

2. En la segunda columna se debe extraer una bola entre las cinco opciones que quedan en la caja. Se tomará un número aleatorio entre 1 y 6 menos el valor extraído en la primera bola:

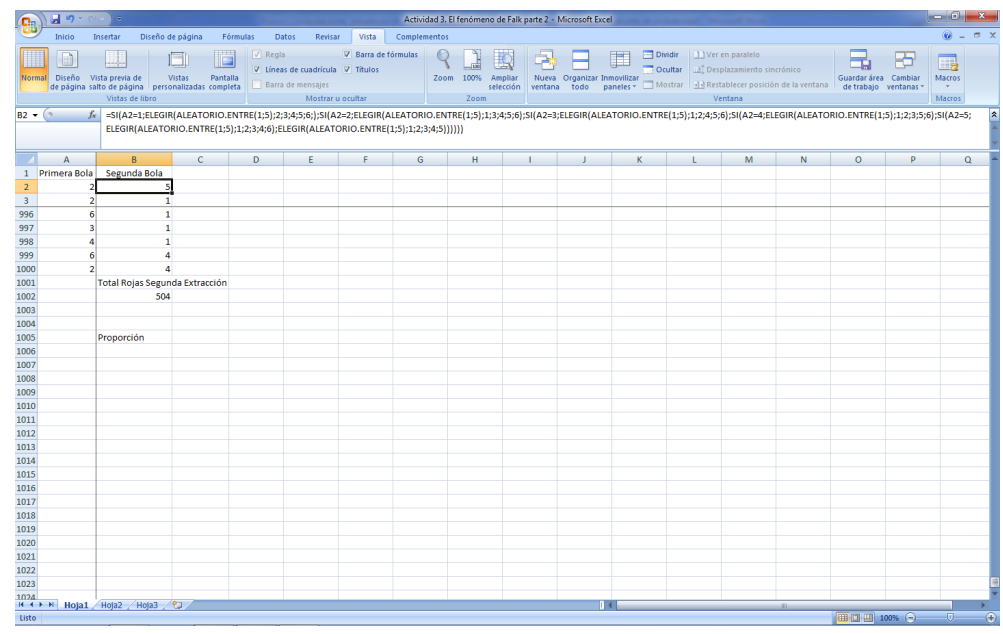

3. Se crea una tercera columna para determinar si ambas bolas extraídas son rojas. Esto es si ambos son valores menores o iguales a tres:

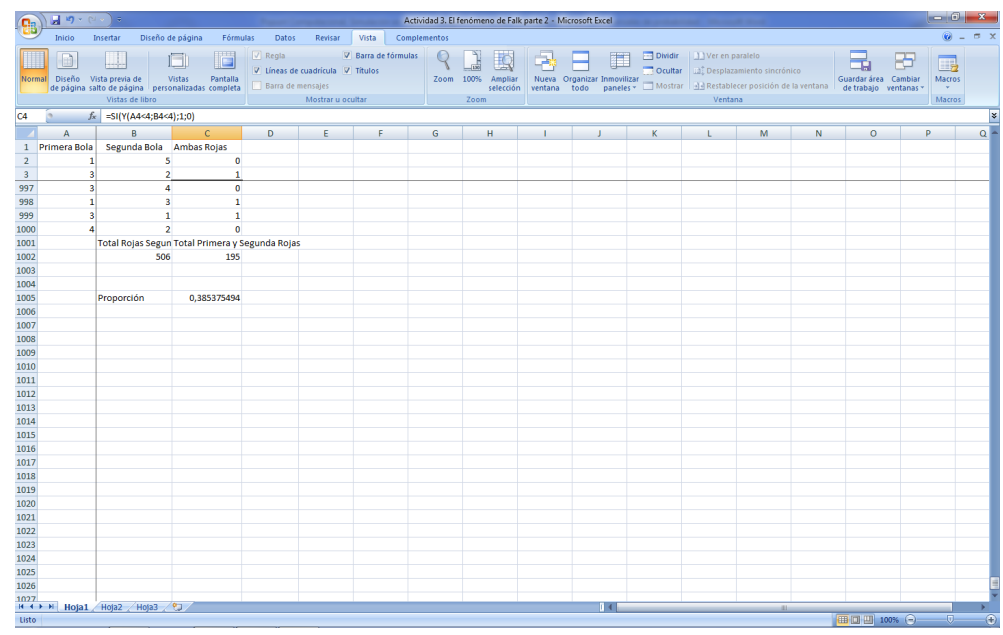


4. Se cuenta, de las 1000 extracciones, la cantidad de veces en las que ambas extracciones son rojas. Además la cantidad de veces en las que la segunda bola fue roja:
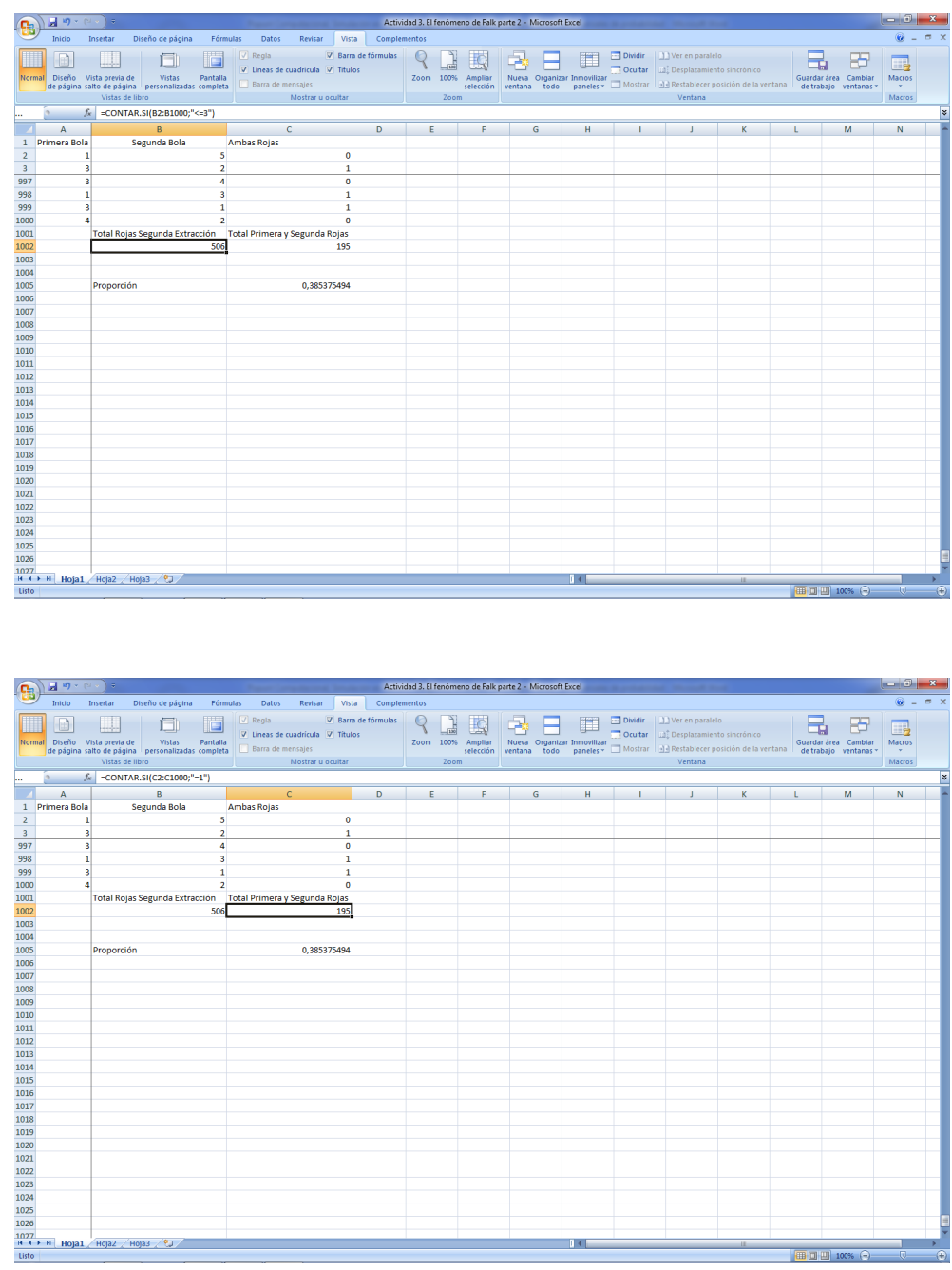

5. Se obtiene la proporción de ambas bolas rojas sobre la cantidad de veces en las que la segunda es roja:

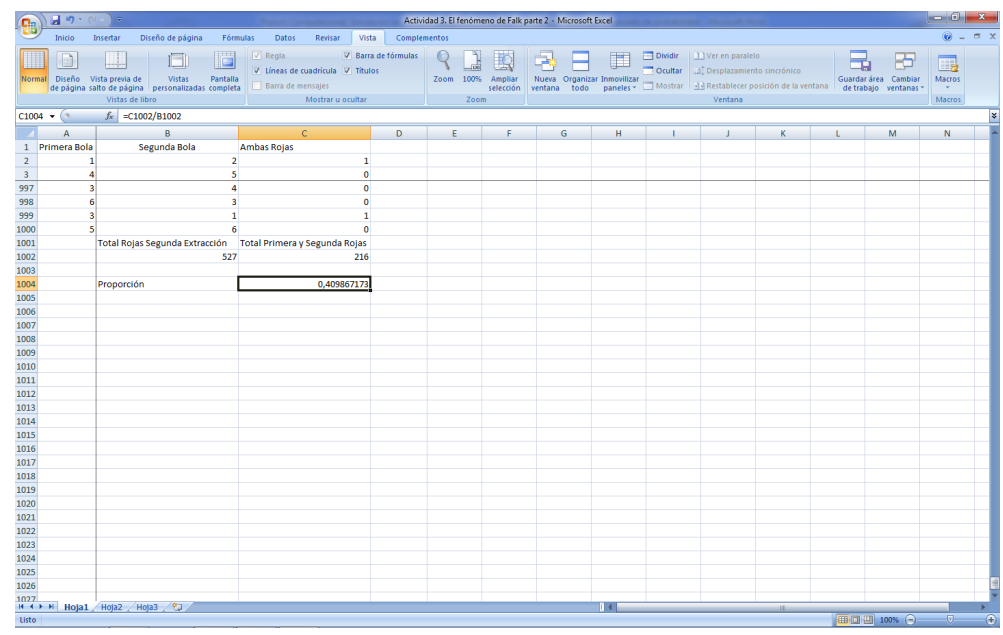


Problema 6. Probabilidad condicional (en Shaughnessy, 1992) (Fathom) Se tienen tres monedas cuyas caras son de colores e igualmente probables de extracción. Una moneda es "blanca" por un lado y "roja" por el otro, otra tiene "rojo" por ambas caras y la otra "blanco" por ambas caras. Si se introducen las monedas en una bolsa y se extrae una al azar sin ver uno de sus lados, ¿qué es más probable con respecto al color que está por el revés de esta misma moneda si el lado visto de la moneda ocurrió que era rojo?

( ) Que sea rojo

( ) Que sea blanco

( ) Son igualmente probables

( ) No se puede determinar

Una propuesta de algoritmo de simulación en Fathom es la siguiente:

1. Se define la caja con las monedas (con los colores y clasificación de las mismas):

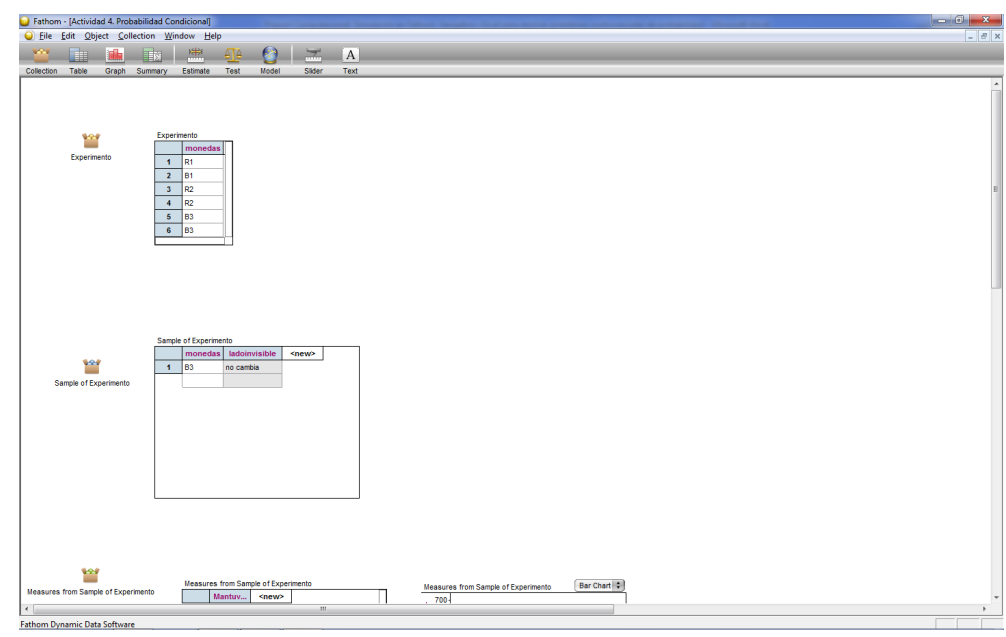

- R1 y B1 significa que es la moneda uno que tiene rojo por un lado y blanco por otro.

- R2 y R2 significa que es la moneda dos que tiene rojo por ambas caras.

- B3 y B3 significa que es la moneda tres que tiene blanco por ambas caras.

2. Se extrae una moneda y observamos el color de una cara. Determinamos si el lado no visto de esa moneda es del mismo color de la cara vista o cambia de color.

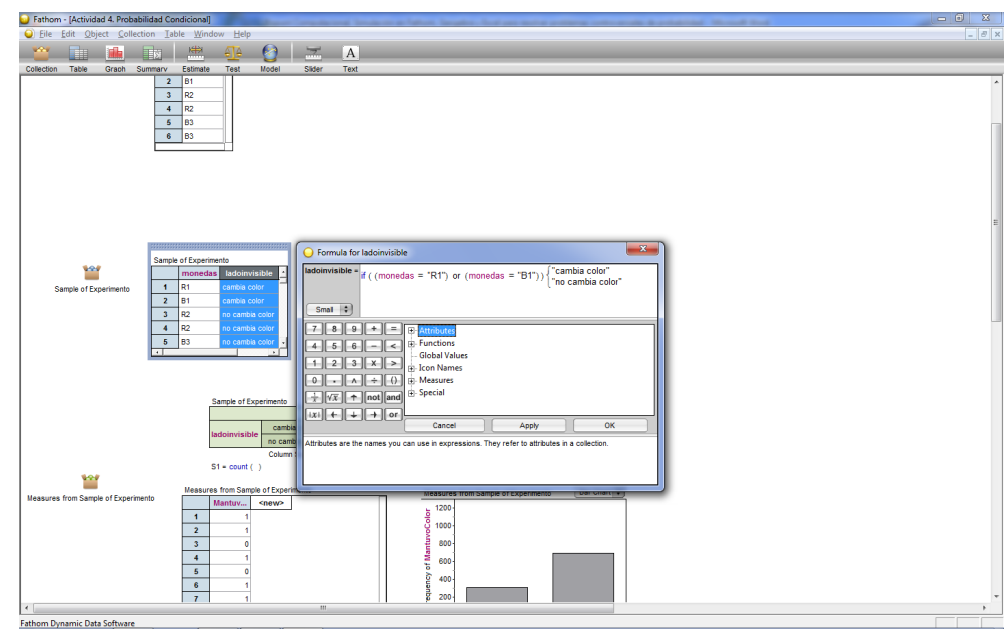


3. Se repite el experimento 1000 veces y se cuanta cuántas veces el lado no visto cambia de color y cuántas veces permanece el mismo color:

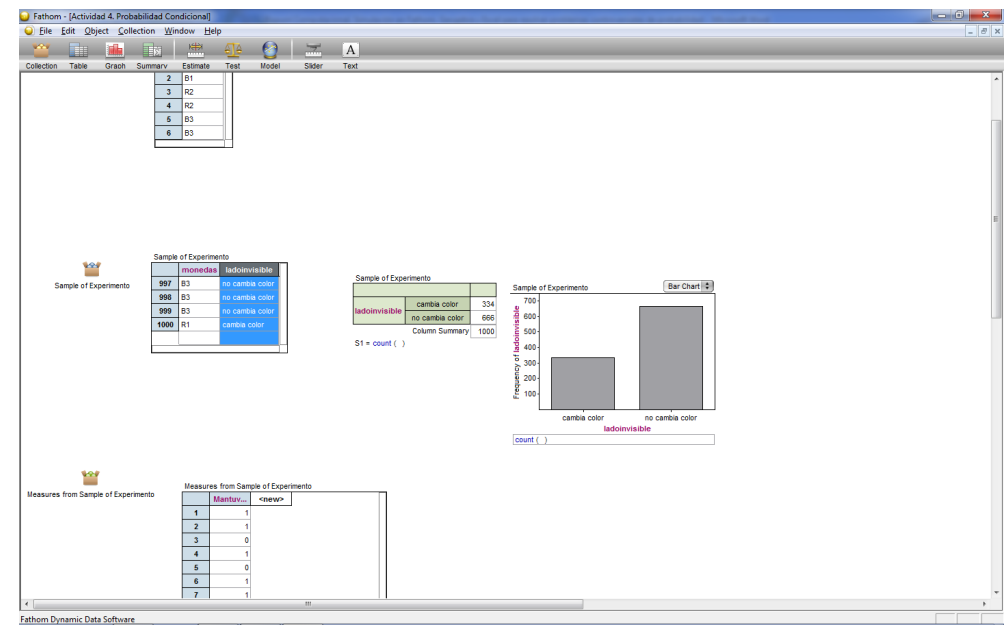

4. Si el lado de una moneda es de cualquier color, ya sea blanco o rojo, es más probable que no cambie de color a su lado inverso. Así que si se sabe que fue rojo en uno de sus lados, es más probable que mantenga el mismo color rojo en su lado inverso. Además, se pueden obtener la proporción de veces que resultó no cambiar de color que es de $\frac{2}{3}$.

\section{Bibliografía}

[1] Batanero, C y Godino J. (2001). Análisis de Datos y su Didáctica. Departamento de Didáctica de la Matemática de la Universidad de Granada.

[2] Burrill, G. (2002). Simulation as a tool to develop statistical understanding. En B. Phillips (Ed). Proceedings of the Sixth International Conference on Teaching Statistics. Cape Town South Africa.

[3] Erickson, T. (2010). Exploring risk though simulation. Reading (Ed.), Data and context in statistics education: Towards an evidence-based society. Proceedings of the Eight International Conference on Teaching Statistics (ICOTS 8). Ljubljana, Slovenia.

[4] Inzunsa, S. (2006). Significados que estudiantes universitarios atribuyen a las distribuciones muestrales en un ambiente de simulación computacional y estadística dinámica. Tesis doctoral no publicada. CINVESTAV-IPN. México.

[5] Lipson, K. (2002). The role of computer based technology in developing understanding of the concept of sampling distribution. En B. Phillips (Ed.). Proceedings of the Sixth International Conference on Teaching Statistics. Cape Town South Africa.

[6] Ministerio de Educación Pública (2005). Programa de Estudio de Matemática: Tercer Ciclo, Costa Rica.

[7] Ministerio de Educación Pública (2005). Programa de Estudio de Matemática: Cuarto Ciclo, Costa Rica.

[8] North, D. Scheiber, J. and Ottaviani, M. (2010). Training teachers to teach statistics in South Africa: realities and attitudes.In C. Reading (Ed.), Data and context in statistics education: Towards an evidence-based society. Proceedings of the Eighth International Conference on Teaching Statistics (ICOTS8, July, 2010), Ljubljana, Slovenia.

[9] Sánchez, E. (2002). Teacher's beliefs about usefulness of simulation with the educational software Fathom for developing probability concepts statistics classroom. En B. Phillips (Ed.). Proceedings of the Sixth International Conference on Teaching Statistics. Cape Town South Africa.

[10] Shaughnessy, M. (1992). Research in Probability and Statistics: Reflections and Directions. En Grouws, D. A.(Ed.). Handbook of Research on Mathematics Teaching and Learning. New York. Macmillan Publishing Company, 465-494. 


\section{Apéndice A}

\section{Anexos}

\section{A.1 actividad 1}

\section{FRECUENCIAS RELATIVAS Y ABSOLUTAS}

\section{Objetivos:}

- Realizar experimentos aleatorios.

- Analizar las frecuencias relativas para experimentos aleatorios.

- Analizar la variabilidad en experimentos aleatorios.

- Comprender las diferencias entre las frecuencias relativas y absolutas en la ley de los grandes números y su efecto en eventos aleatorios.

\section{Actividades.}

1. Cree una colección llamada Experimento, denote al atributo con el nombre de moneda y cree manualmente los casos E (escudo) y H (corona).

2. Tome una muestra con reemplazo de la colección Experimento de tamaño 4. Debe utilizar el comando Sample Cases del menú Collection. 
3. En este momento nos interesa contar cuántas ocasiones ocurrió E (Escudo) Utilizando la pestaña Measures del Inspect Sample y el comando Count, determine cuántas veces moneda = "E".

4. Repita este experimento 1000 veces. Esto es realizar una colección de medidas con el comando Collect of Measure sobre la colección aleatoria obtenida.

5. Construya un histograma con los resultados obtenidos en el punto anterior. ¿Cómo describiría usted la gráfica obtenida?

6. Construya ahora un diagrama de caja con los mismos datos, ¿cómo explicaría usted el comportamiento de la gráfica?

7. Determine, de esos 1000 experimentos, cuántas veces se obtuvo exactamente 2 escudos. Esto es utilizando una tabla Summary y arrastrando el atributo a la tabla y dejando la tecla Shift presionada

8. ¿Cuál es el valor de la desviación estándar de la variable obtenida?

9. Repita nuevamente desde al paso 2 hasta el paso 8, pero tomando muestras de tamaño 100 y averiguando de los 1000 experimentos, cuántas veces se obtuvo exactamente 50 escudos.

10. Con base en el punto 7 y 8 , ¿cuál de los siguientes eventos considera que es más probable?

- ( ) Obtener dos escudos en cuatro intentos

- ( ) Obtener 50 escudos en 100 intentos

- ( ) Los dos anteriores son igualmente probables

¿Por qué crees que sucede esto?

11. Con base en el punto 6, ¿en cuál distribución hay mayor variabilidad, en la de 4 lanzamientos o en la de 100 lanzamientos?

¿Por qué crees que sucede esto?

\section{A.2 ACTIVIDAD 2}

\section{PROBLEMA DEL CÁNCER}

\section{Objetivos:}

- Realizar experimentos aleatorios.

- Analizar las frecuencias relativas para experimentos aleatorios.

- Analizar la variabilidad en experimentos aleatorios.

- Comprender la probabilidad condicional como frecuencia relativa.

\section{Actividades.}

1. Cree una variable llamada AleTieneCancer, a la cual se le define un random de 1000 valores en el intervalo ]0,1]. Esto es utilizamos el comando: AleTieneCancer = Secuencia [random(), i, 1, 1000] 
2. Cambie las opciones para que se trabaje con 4 decimales. Esto es del menú Opciones, en la opción Redondeo, selecciones trabajar con $\{4$ Lugares decimales.

3. Definamos la variable TieneCancer, que se le define la probabilidad para una mujer de tener cáncer según los valores de la variable AleTieneCancer anterior.Así: TieneCancer = Secuencia[Si[Elemento[AleTieneCancer, i] $\leqslant 0.008$, "C", "NoC"], i, 1, 1000]

4. Ahora cree la variable AleMamoconCan, a la cual se le define un random de 1000 valores en el intervalo $] \mathbf{0 , 1}]$. Esto es: AleMamoconCan = Secuencia $[$ random(), i, 1, 1000]

5. En forma similar se crea la variable AleMamosincan, a la cual se le define un random de 1000 valores en el intervalo ]0,1]. Esto es: AleMamosinCan = Secuencia[random(), i, 1, 1000]

6. Definamos la variable MamoconsinCan, que se le define la probabilidad para una mujer de que la mamografía sea positiva según los valores de las variables AleMamoconCan y AleMamosinCan anterior. Así: MamoconsinCan = Secuencia[Si[Elemento[TieneCancer, i] $\stackrel{?}{=}$ "NoC", Si[Elemento[AleMamosinCan, i] <0.07, "MP", "MN"], Si[Elemento[AleMamoconCan, i] <0.1, "MN", "MP"]], i, 1, 1000]

7. Ahora obtenemos el total de mamografías positivas, para ellos definimos la variable TotalmP y le asignamos: TotalMP =Secuencia [Si[Elemento [MamoconsinCan, i] $\stackrel{?}{=}$ "MP", 1, 0], i, 1, 1000]

8. Además, obtenemos el total de veces que se tuvo cáncer y a la vez sus mamografías resultaron positivas, para ellos definimos la variable TotalTieneCanceryMP y le asignamos:

TotalTieneCanceryMP =Secuencia[Si[Elemento[TieneCancer, i] $\stackrel{?}{=} C^{\prime \prime} \wedge$ Elemento[MamoconsinCan, i] $\stackrel{?}{=}$ "MP", 1, 0], i, 1, 1000]

9. Por último contamos para las variables de los dos últimos pasos cuántos unos obtenemos respectivamente. Creamos las variables $a$ y $b$ y les asignamos:

$$
\begin{aligned}
& a=\text { CuentaSi }[\mathrm{x} \stackrel{?}{=} 1, \text { TotalTieneCanceryMP }] \\
& b=\text { CuentaSi }[\mathrm{x} \stackrel{?}{=} 1, \text { TotalMP }]
\end{aligned}
$$

10. Obtenemos la frecuencia relativa de veces en las que resulto tener cáncer y su mamografía fue positiva con respecto al total de veces en la que la mamografía resultó positiva. Finalmente: $c=a / b$

11. Entonces, si una mujer decide hacerse una mamografía y el resultado es positivo, ¿cuál es la probabilidad de que la mujer tenga cáncer?

\section{A.3 actividad 3 .}

\section{EL FENÓMENO DE FALK. I Parte}

\section{Objetivos:}

- Realizar experimentos aleatorios.

Un taller de simulaciones: Fathom, GeoGebra y Excel para resolver problemas controversiales de probabilidad. Greivin Ramírez Arce. Derechos Reservados (C) 2012 Revista digital Matemática, Educación e Internet (www.tec-digital.itcr.ac.cr/revistamatematica/) 
- Analizar la probabilidad condicional de eventos causales y eventos condicionados a priori.

\section{Actividades.}

1. Cree una variable llamada Primera_Bola, a la cual se le define un random de 1000 valores en el intervalo entero[1,3]. Esto es utilizamos el comando: =ALEATORIO.ENTRE $(1 ; 3)$

2. Cree otra variable llamada Segunda_Bola, a la cual se le define un random de 1000 valores en el intervalo entero $[\mathbf{1 , 6}]$. Así:

$=\operatorname{SI}(A 2=1 ; \operatorname{ELEGIR}($ ALEATORIO.ENTRE $(1 ; 5) ; 2 ; 3 ; 4 ; 5 ; 6 ;) ; \operatorname{SI}(\mathrm{A} 2=2 ;$

ELEGiR (ALEATORIO. ENTRE $(1 ; 5) ; 1 ; 3 ; 4 ; 5 ; 6) ; \operatorname{ELEGIR}(\operatorname{AlEATORIO}$. ENTRE $(1 ; 5) ; 1 ; 2 ; 4 ; 5 ; 6))$ )

3. Se obtiene, de los 1000 valores, la proporción de extracciones en las que la segunda bola fue roja dado que la primera fue roja. Así: Proporción de rojas =CONTAR.SI (B2:B1000;"<=3") /1000

4. Una urna tiene en su interior tres bolas rojas y tres bolas azules. Se extraen dos bolas sin reemplazar la primera. ¿Cuál es la probabilidad de que la segunda bola sea roja dado que la primera fue roja?

\section{A.4 ACTIVIDAd 3.}

\section{EL FENÓMENO DE FALK. II Parte}

\section{Objetivos:}

- Realizar experimentos aleatorios.

- Analizar la probabilidad condicional de eventos causales y eventos condicionados a priori.

\section{Actividades.}

1. En un nuevo archivo, cree una variable llamada Primera_Bola, a la cual se le define un random de 1000 valores en el intervalo entero $[\mathbf{1}, \mathbf{6}]$. Esto es utilizamos el comando: $=\operatorname{ALEATORIO} . \operatorname{ENTRE}(1 ; 6)$

2. Cree otra variable llamada Segunda_Bola, a la cual se le define un random de 1000 valores en el intervalo entero $[\mathbf{1}, 6]$ - Primera_Bola. Así:

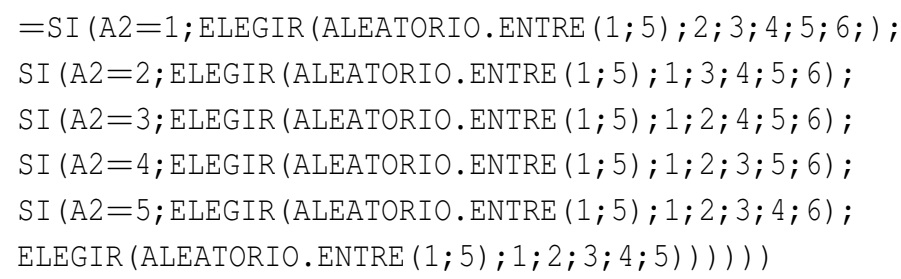

3. Se obtiene, de los 1000 valores de la segunda extracción, la cantidad total de bolas que resultaron ser rojas. Esto es: Total Rojas Segunda Extracción = CONTAR.SI (B2:B1000;"<=3")

4. Se define una nueva variable llamada Ambas_Rojas, que será la que contabilice las ocasiones en las que en ambas extracciones resultaron bolas rojas. Para cada pareja de extracciones se le asigna a esta variable un "1" si ambas resultaron ser rojas o un " 0 " en caso contrario. Así: $=\mathrm{SI}(\mathrm{Y}(\mathrm{A} 2<4 ; \mathrm{B} 2<4) ; 1 ; 0)$ 
5. Se obtiene, de los 1000 valores anteriores, la cantidad total de parejas en las que ambas extracciones resultaron ser rojas. Esto es: Total Primera y Segunda Rojas = CONTAR.SI (C2:C1000;"=1")

6. Se obtiene, de los 1000 valores, la proporción de extracciones en las que la primera bola fue roja dado que la segunda fue roja. Así:

$$
\text { Proporción }=\frac{\text { Total Primera y Segunda Rojas }}{\text { Total Rojas Segunda Extracción }}
$$

7. Una urna tiene en su interior tres bolas rojas y tres bolas azules. Se extraen dos bolas sin reemplazar la primera. ¿Cuál es la probabilidad de que la primera bola sea roja, dado que la segunda es roja?

\section{A.5 Actividad 4}

\section{PROBABILIDAD CONDICIONAL.}

\section{Objetivos:}

- Realizar experimentos aleatorios.

- Analizar la variabilidad en experimentos aleatorios.

- Utilizar simulación y el cálculo de frecuencias relativas para el cálculo intuitivo de probabilidades

1. Cree una colección llamada Experimento, denote al atributo con el nombre de monedas y cree manualmente los casos R1 (rojo moneda 1), B1 (blanco moneda 1), R2 (rojo moneda 2), R2 (rojo moneda 2), B3 (blanco moneda 3) y B3 (blanco moneda 3).

2. Tome una muestra con reemplazo de la colección Experimento de tamaño 1.

3. Cree un nuevo atributo llamado lado invisible y utilice el condicional de que si sale una moneda con igual color en ambas caras entonces el color se mantiene en el dorso de la moneda, sino el color cambia.

4. Luego cuente cuántas veces el color se mantuvo. Utilizando la pestaña Measures del Inspect Sample y el comando count.

5. Repita este experimento 1000 veces. Esto es realizar una colección de medidas con el comando collect of Measure sobre la colección aleatoria obtenida.

6. Construya un gráfico de barras con los resultados obtenidos en el punto anterior. Esto es arrastrando el atributo al gráfico y dejando la tecla Shift presionada. ¿Cómo describiría usted la gráfica obtenida?

7. Determine, de esos 1000 experimentos, la frecuencia relativa del número de veces que cambia de color el dorso de la moneda y cuántas veces se mantiene. Esto es utilizando una tabla Summary y arrastrando el atributo a la tabla y dejando la tecla Shift presionada

8. Se tienen tres monedas cuyas caras son de colores e igualmente probables de extracción. Una moneda es "blanca" por un lado y "roja" por el otro, otra tiene "rojo" por ambas caras y la otra "blanco" por ambas caras.

Si se introducen las monedas en una bolsa y se extrae una al azar sin ver uno de sus lados, ¿qué es más probable con respecto al color que está por el revés de esta misma moneda si el lado visto de la moneda ocurrió que era rojo? 
( ) Que sea rojo

( ) Que sea blanco

( ) Son igualmente probables

( ) No se puede determinar

Un taller de simulaciones: Fathom, GeoGebra y Excel para resolver problemas controversiales de probabilidad. Greivin Ramírez Arce. Derechos Reservados (C) 2012 Revista digital Matemática, Educación e Internet (www.tec-digital.itcr.ac.cr/revistamatematica/) 